\title{
Thermal Shock Problem of Generalized Thermoelasticity for an Infinitely Long Annular Cylinder with Variable Thermal Conductivity
}

\author{
H. M. Youssef ${ }^{1 *}$ and I. A. Abbas ${ }^{2}$ \\ ${ }^{1}$ Mathematical Department, Faculty of Education \\ Alexandria University, Alexandria, Egypt \\ e-mail:yousefanne@yahoo.com \\ ${ }^{2}$ Department of Mathematics, Faculty of Science \\ Sohag University 82524, Egypt \\ e-mail: ibrabbas7@yahoo.com
}

(Rec. May 17, 2007)

\begin{abstract}
In this paper, a general finite element model is proposed to analyze transient phenomena in thermoelastic model in the context of the theory of generalized thermoelasticity with one relaxation time with variable thermal conductivity. An application of an infinitely long annular cylinder was studied, where the inner surface is traction free and subjected to thermal shock, while the outer surface is traction free and thermally isolated. The results for the temperature increment, the stress components and the displacement component are illustrated graphically.
\end{abstract}

Key words: thermoelasticity, generalized thermoelasticity, annular cylinder, finite element

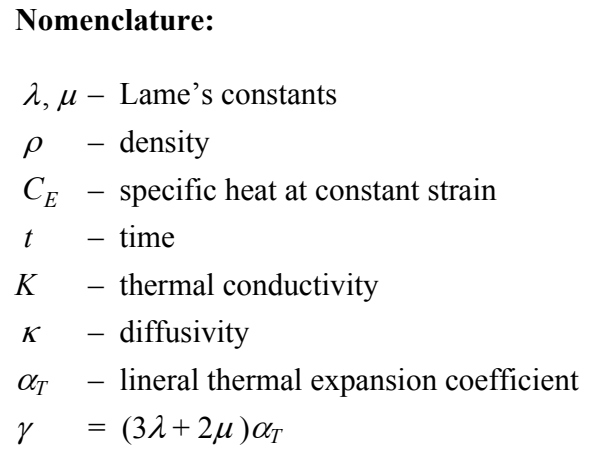

\section{INTRODUCTION}

The classical uncoupled theory of thermoelasticity predicts two phenomena not compatible with physical observations. First, the equation of heat conduction of this theory does not contain any elastic terms. Second, the heat equation is of a parabolic type, predicting infinite speeds of propagation for heat waves.

Biot [1] introduced the theory of coupled thermoelasticity to overcome the first shortcoming. The governing

$$
\begin{aligned}
& T-\text { absolute temperature } \\
& T_{0}-\text { reference temperature } \\
& \theta-\text { temperature increment } \theta=\left|T-T_{0}\right| \\
& \vartheta \quad \text { - the mapping of } \theta \\
& \sigma_{i j}-\text { components of stress tensor } \\
& e_{i j}-\text { components of strain tensor } \\
& u_{i}-\text { components of displacement vector } \\
& \tau_{0}-\text { relaxation time }
\end{aligned}
$$


ralized thermoelasticity are being extensively used-one proposed by Lord and Shulman [2] and the other proposed by Green and Lindsay [3]. L-S (Lord and Shulman theory) suggests one relaxation time and according to this theory, only Fourier's heat conduction equation is modified, while G-L (Green and Lindsay theory) suggests two relaxation times and both the energy equation and the equation of motion are modified.

Eraby and Suhubi [4] studied wave propagation in a cylinder. Ignaczak [5] studied a strong discontinuity wave and obtained a decomposition theorem [6]. Ezzat [7] has also obtained the fundamental solution for this theory. Many problems have been solved in the context of the generalized thermoelasticity by Youssef et al. [8-13].

Modern structural elements are often subjected to temperature changes of such magnitude that their material properties may no longer be regarded as having constant values even in an approximate sense. The thermal and mechanical properties of materials vary with temperature, so that the temperature dependence of material properties must be taken into consideration in the thermal stress analysis of these elements $[14,15]$.

In this work, we will construct a model of theory of generalized thermoelasticity with one relaxation time considering the thermal conductivity to be variable. We consider an infinitely long annular cylinder whose inner surface is traction free and subjected to thermal shock. The outer surface is also traction free and thermally isolated. The medium parameters quiescent initial state. a general finite element model is proposed to get the solution and the results are represented graphically.

\section{THE GOVERNING EQUATIONS}

The heat equation [19]:

$$
\left(K \theta_{, i}\right)_{, i}=\left(1+\tau_{0} \frac{\partial}{\partial t}\right)\left[\rho C_{E} \dot{\theta}+\gamma T_{0} \dot{e}\right] \quad i=1,2,3,
$$

which can be written in the form

$$
\left(K \theta_{, i}\right)_{, i}=\left(1+\tau_{0} \frac{\partial}{\partial t}\right)\left[\frac{K}{\kappa} \dot{\theta}+\gamma T_{0} \dot{e}\right],
$$

where

$$
\rho C_{E}=\frac{K}{\kappa}, \quad \theta=\left|T-T_{0}\right|,
$$

$K$ is called the thermal conductivity ( $K_{1}$ is a small value) and $\kappa$ is the diffusivity (assumed to be constant).

We will use the mapping [16]:

$$
\vartheta=\frac{1}{K_{0}} \int_{0}^{\theta} K\left(\theta^{\prime}\right) d \theta^{\prime}
$$

By differentiating the last mapping with respect to $x_{i}$, we get

$$
K_{0} \vartheta_{, i}=K(\theta) \theta_{, i}
$$

By differentiating the last equation again with respect to $x_{i}$, we get

$$
K_{0} \vartheta_{, i i}=\left[K(\theta) \theta_{, i}\right]_{, i}
$$

With the same manner, by differentiating the mapping with respect to time, we have

$$
K_{0} \dot{\vartheta}=K(\theta) \dot{\theta}
$$

Hence, the heat equation will take the form

$$
\vartheta_{, i i}=\left[\frac{\partial}{\partial t}+\tau_{0} \frac{\partial^{2}}{\partial t^{2}}\right]\left[\frac{\vartheta}{\kappa}+\frac{\gamma T_{0}}{K_{0}} e\right]
$$

Now, we will take the thermal conductivity as a function of the temperature with linear form as follows [16]:

$$
K=K(\theta)=K_{0}\left(1+K_{1} \theta\right)
$$

Then, we have from the last equation and the mapping the following forms

$$
\begin{gathered}
\vartheta=\theta+\frac{K_{1}}{2} \theta^{2}, \\
\vartheta_{, i}=\theta_{, i}\left(1+K_{1} \theta\right)
\end{gathered}
$$

and

$$
\theta=\frac{-1+\sqrt{1+2 K_{1} \vartheta}}{K_{1}}
$$

Now, we have the equations of motion in the form [16]:

$$
\rho \ddot{u}_{i}=(\lambda+\mu) u_{j, j i}+\mu u_{i, j j}-\gamma \theta_{, i}
$$

which can be written as follows

$$
\rho \ddot{u}_{i}=(\lambda+\mu) u_{j, j i}+\mu u_{i, j j}-\gamma \frac{\vartheta_{, i}}{\left(1+K_{1} \theta\right)} .
$$

From the relation (11) we have

$$
1+K_{1} \theta=\sqrt{1+2 K_{1} \vartheta} .
$$

Then, we have

$$
\rho \ddot{u}_{i}=(\lambda+\mu) u_{j, j i}+\mu u_{i, j j}-\gamma \frac{\vartheta_{, i}}{\sqrt{1+2 K_{1} \vartheta}} .
$$

The constitutive relation take the form [16]: 


$$
\sigma_{i j}=2 \mu e_{i j}+\left(\lambda e_{k k}-\gamma \frac{-1+\sqrt{1+2 K_{1} \vartheta}}{K_{1}}\right) \delta_{i j} .
$$

\section{FORMULATION OF THE PROBLEM}

We consider an infinitely long annular cylinder whose inner surface is traction free and subjected to a thermal shock, while the outer surface also is traction free but thermally isolated. We assume also that there are no external body forces or heat sources acting in the medium.

We use a cylindrical system of coordinates $(r, \psi, z)$ with the $z$-axis lying along the axis of the cylinder.

Due to symmetry, the problem is one-dimensional with all the functions considered depending on the radial distance $r$ and the time $t$ where $R_{1} \leq r \leq R_{2}$.

The displacement vector has the components

$$
u_{r}=u(r, t), \quad u_{\psi}(r, t)=u_{z}(r, t)=0 .
$$

From equation (7), the heat equation takes the form

$$
\nabla^{2} \vartheta=\left[\frac{\partial}{\partial t}+\tau_{0} \frac{\partial^{2}}{\partial t^{2}}\right]\left[\frac{v}{\kappa}+\frac{\gamma T_{0}}{K_{0}} e\right]
$$

where

$$
\nabla^{2}=\frac{\partial^{2}}{\partial r^{2}}+\frac{1}{r} \frac{\partial}{\partial r}
$$

from equation (16), the equation of motion has the form

$$
\rho \ddot{u}=(\lambda+2 \mu) \frac{\partial e}{\partial r}-\frac{\gamma}{\sqrt{1+2 K_{1} \vartheta}} \frac{\partial \vartheta}{\partial r},
$$

where

$$
e=\frac{1}{r} \frac{\partial(r u)}{\partial r}
$$

and from equation (16), the constitutive equations take the forms

$$
\begin{gathered}
\sigma_{r r}=2 \mu \frac{\partial u}{\partial r}+\lambda e-\gamma\left(\frac{-1+\sqrt{1+2 K_{1} \vartheta}}{K_{1}}\right), \\
\sigma_{\psi \psi}=2 \mu \frac{u}{r}+\lambda e-\gamma\left(\frac{-1+\sqrt{1+2 K_{1} \vartheta}}{K_{1}}\right), \\
\sigma_{z z}=\lambda e-\gamma\left(\frac{-1+\sqrt{1+2 K_{1} \vartheta}}{K_{1}}\right), \\
\sigma_{z r}=\sigma_{\psi r}=\sigma_{z z}=0 .
\end{gathered}
$$

We will use the following non-dimensional variables

$$
\begin{gathered}
r^{\prime}=\left(\frac{\lambda+2 \mu}{\rho}\right)^{1 / 2} \frac{r}{\kappa}, \quad u^{\prime}=\left(\frac{\lambda+2 \mu}{\rho}\right)^{1 / 2} \frac{u}{\kappa}, \\
t^{\prime}=\left(\frac{\lambda+2 \mu}{\rho}\right) \frac{t}{\kappa} \quad \tau_{0}^{\prime}=\left(\frac{\lambda+2 \mu}{\rho}\right) \frac{\tau_{0}}{\kappa}, \\
q^{\prime}=\frac{\kappa}{K_{0} T_{0}}\left(\frac{\rho}{\lambda+2 \mu}\right)^{1 / 2} q, \quad R^{\prime}=\left(\frac{\lambda+2 \mu}{\rho}\right)^{1 / 2} \frac{R}{\kappa}, \\
\vartheta^{\prime}=\frac{\vartheta}{T_{0}}, \quad \sigma^{\prime}=\frac{\sigma}{\mu}, \quad K_{1}^{\prime}=K_{1} T_{0} .
\end{gathered}
$$

Using these non-dimensional variables, the above equations take the form (dropping the primes for convenience)

$$
\nabla^{2} \vartheta=\left[\frac{\partial}{\partial t}+\tau_{0} \frac{\partial^{2}}{\partial t^{2}}\right][\vartheta+g e]
$$

$$
\ddot{e}=\nabla^{2} e-\frac{a}{\sqrt{1+2 K_{1} \vartheta}} \nabla^{2} \vartheta+\frac{a K_{1}}{\left(1+2 K_{1} \vartheta\right)^{3 / 2}}\left(\frac{\partial \vartheta}{\partial r}\right)^{2}
$$

$$
\sigma_{r r}=\beta^{2} \frac{\partial u}{\partial r}+\left(\beta^{2}-2\right) \frac{u}{r}-b\left(\frac{-1+\sqrt{1+2 K_{1} \vartheta}}{K_{1}}\right),
$$

$$
\sigma_{\psi \psi}=\left(\beta^{2}-2\right) \frac{\partial u}{\partial r}+\beta^{2} \frac{u}{r}-b\left(\frac{-1+\sqrt{1+2 K_{1} \vartheta}}{K_{1}}\right)
$$

$$
\sigma_{z z}=\left(\beta^{2}-2\right) e-b\left(\frac{-1+\sqrt{1+2 K_{1} \vartheta}}{K_{1}}\right)
$$

where

$$
b=\frac{\gamma T_{0}}{\mu}, \quad g=\frac{\gamma \kappa}{K_{0}}, \quad \beta=\left(\frac{\lambda+2 \mu}{\mu}\right)^{1 / 2} \quad \text { and } \quad a=\frac{b}{\beta^{2}} .
$$

We will use the boundary conditions on the internal surface, $r=R_{1}$ and the outer surface, $r=R_{2}$ which are given by

(1) The thermal boundary conditions

I. The internal surface $r=R_{1}$ is subjected to a thermal shock in the form

$$
\begin{aligned}
& \theta(R, t)=\theta_{0} H(t), \\
& \vartheta\left(R_{1}, t\right)=\delta H(t),
\end{aligned}
$$

where

$$
\delta=\left(1+\frac{K_{1}}{2} \theta_{0}\right) \theta_{0}
$$


II. The outer surface $r=R_{2}$, we have not any heat flux. We will use the generalized Fourier law of heat conduction, namely

$$
q_{r}+\tau_{0} \frac{\partial q_{r}}{\partial t}=-K(\theta) \frac{\partial \theta}{\partial r}
$$

By using equation (4), we have

$$
q_{r}+\tau_{0} \frac{\partial q_{r}}{\partial t}=-K_{0} \frac{\partial \vartheta}{\partial r}
$$

After using the non-dimensional variables, the last equation will take the form

$$
q_{r}+\tau_{0} \frac{\partial q_{r}}{\partial t}=-\frac{\partial \vartheta}{\partial r}
$$

Now, by using the boundary condition at $r=R_{2}$ which we have $q_{r}=0$.

Then, we get

$$
\frac{\partial \bar{\vartheta}\left(R_{2}, s\right)}{\partial r}=0 .
$$

(2) The mechanical boundary conditions

The internal and the outer surfaces $r=R_{1}$ and $r=R_{2}$ is traction free i.e.

$$
\sigma_{r r}\left(R_{1}, t\right)=0
$$

and

$$
\sigma_{r r}\left(R_{2}, t\right)=0 \text {. }
$$

\section{FINITE ELEMENT METHOD}

In order to investigate the thermo-mechanical shock problem of generalized thermoelasticity for an infinitely long annular cylinder with variable thermal conductivity problem by finite element method, the (FEM) [17-19] is adopted due to its flexibility in modeling layered structures and its capability in obtaining full field numerical solution. The governing equations (25) and (26) are coupled with initial and boundary conditions. The numerical values of the dependent variables like displacement $u$ and the mapping of temperature $\vartheta$ are obtained at the interesting points which are called degrees of freedom. The weak formulations of the nondimensional governing equations are derived. The set of independent test functions to consist of the displacement $\delta u$ and the mapping of temperature $\delta \vartheta$ is prescribed. The governing equations are multiplied by independent weighting functions and then are integrated over the spatial domain with the boundary. Applying integration by parts and making use of the divergence theorem reduce the order of the spatial derivatives and allows for the application of the boundary conditions. The same shape functions are defined piecewise on the elements. Using the Galerkin procedure, the unknown fields $u$ and $\vartheta$ and the corresponding weighting functions are approximated by the same shape functions. The last step towards the finite element discretization is to choose the element type and the associated shape functions. Three nodes of quadrilateral elements are used. The shape function is usually denoted by the letter $N$ and is usually the coefficient that appears in the interpolation polynomial. A shape function is written for each individual node of a finite element and has the property that its magnitude is 1 at that node and 0 for all other nodes in that element. We assume that the master element has its local coordinates in the range $[-1,1]$. In our case, the one-dimensional quadratic elements are used, which given by:

linear shape functions

$$
N_{1}=\frac{1}{2}(1-\xi), \quad N_{2}=\frac{1}{2}(1+\xi),
$$

quadratic shape functions

$$
N_{1}=\frac{1}{2}\left(\xi^{2}-\xi\right), \quad N_{2}=1-\xi^{2}, \quad N_{3}=\frac{1}{2}\left(\xi^{2}+\xi\right) .
$$

On the other hand, the time derivatives of the unknown variables have to be determined by Newmark time integration method with 0.02 as time step [17]. In our investigation, we prepared the programs for finite element methods by using Scilab and Matlab software.

After obtaining $\vartheta$, the temperature increment $\theta$ can be obtained by solving equation (11).

\section{NUMERICAL RESULTS AND DISCUSSION}

The copper material was chosen for purposes of numerical evaluations. The constants of the material were taken as [13]:

$$
\begin{gathered}
K_{0}=386 \mathrm{~km} \mathrm{~m} \mathrm{~K}^{-1} \mathrm{~s}^{-3}, \quad \alpha_{T}=1.78(10)^{-5} \mathrm{~K}^{-1}, \\
\rho=8954 \mathrm{~kg} \mathrm{~m}^{-3}, \quad T_{0}=293 \mathrm{~K}, \quad C_{E}=383.1 \mathrm{~m}^{2} \mathrm{~K}^{-1} \mathrm{~s}^{-2} \\
\mu=3.86(10)^{10} \mathrm{~kg} \mathrm{~m}^{-1} \mathrm{~s}^{-2}, \quad \lambda=7.76(10)^{10} \mathrm{~kg} \mathrm{~m}^{-1} \mathrm{~s}^{-2}, \\
\tau_{0}=0.02 \mathrm{~s}, \quad \beta^{2}=4, \quad b=0.042, \quad g=1.61, \quad a=0.0105 .
\end{gathered}
$$

The computations were carried out for $t=0.20, R_{1}=1$, $R_{2}=3$ and $\theta_{0}=1$ with different values of $K_{1}(0.0,-0.5$, $-1.0)$ where the value of $K_{1}=0.0$ shows the old case when 
the thermal conductivity is independent of temperature. The field quantities, temperature, stresses and displacement depend not only on the state and space variables $t$ and $r$ but also depend on the value of $K_{1}$. It has been observed that, $K_{1}$ plays a vital role on the development of all the fields.

Figure 1, displays the temperature distribution and we have noticed that, the value of $K_{1}$ has a significant effect on the temperature. In the same point of $r$, when $K_{1}$ decreases, the temperature decreases.

Figure 2, displays the displacement distribution and we have noticed that, the value of $K_{1}$ has a significant effect on the displacement. In the same point of $r$, when $K_{1}$ decreases, the absolute value of the displacement decreases.

Figures 3 and 4, displays the stresses distribution and we have noticed that, the value of $K_{1}$ has a significant effect on the stresses. In the same point of $r$, when $K_{1}$ decreases, the absolute value of the stresses decreases.

Physically, we can say that, when $K$ is variable with linear function of temperature with negative values of $K_{1}$, the values of the thermal conductivity decreasing with increasing temperature and then the distance between the particles will increase which makes the speed of waves

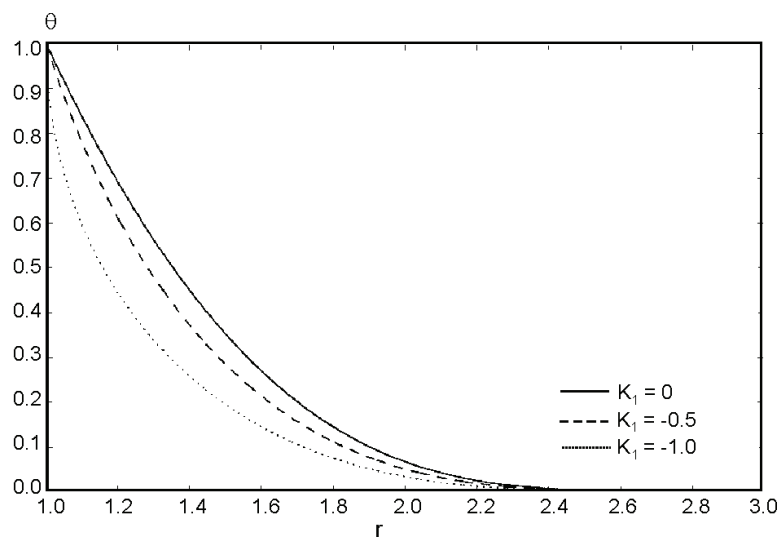

Fig. 1. The temperature distribution with different values of $K_{1}$

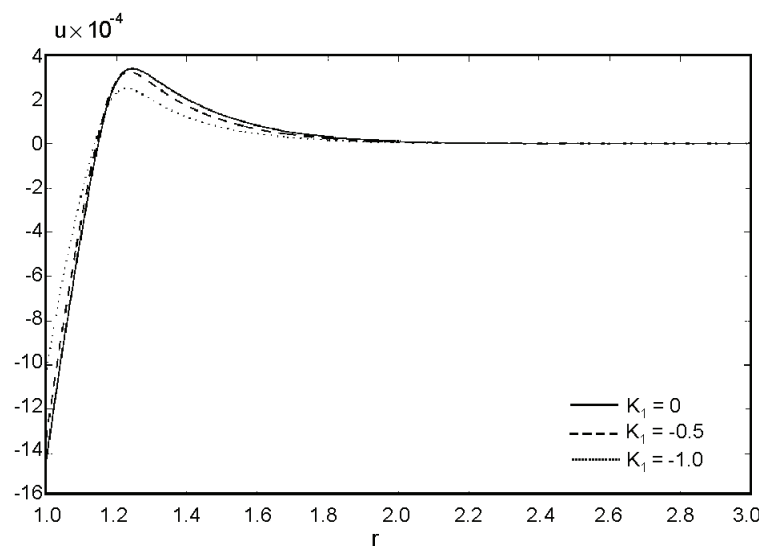

Fig. 2. The displacement distribution with different values of $K_{1}$

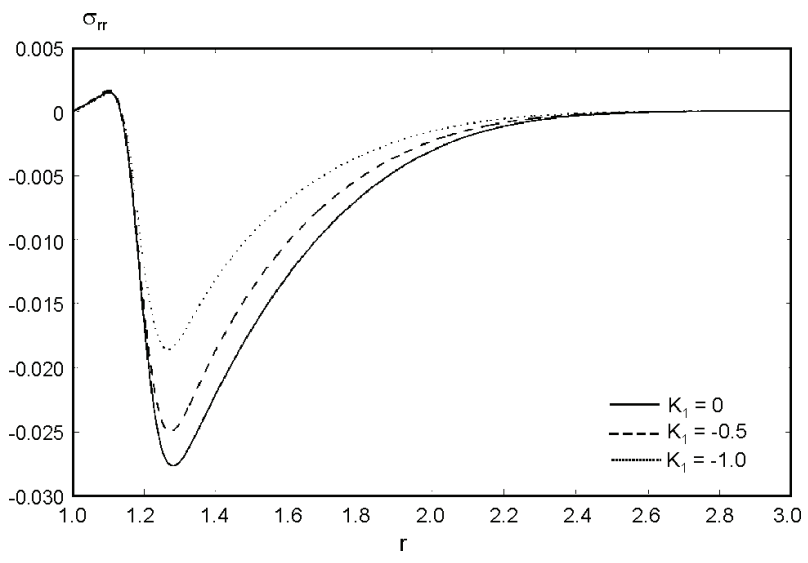

Fig. 3. The stress distribution with different values of $K_{1}$

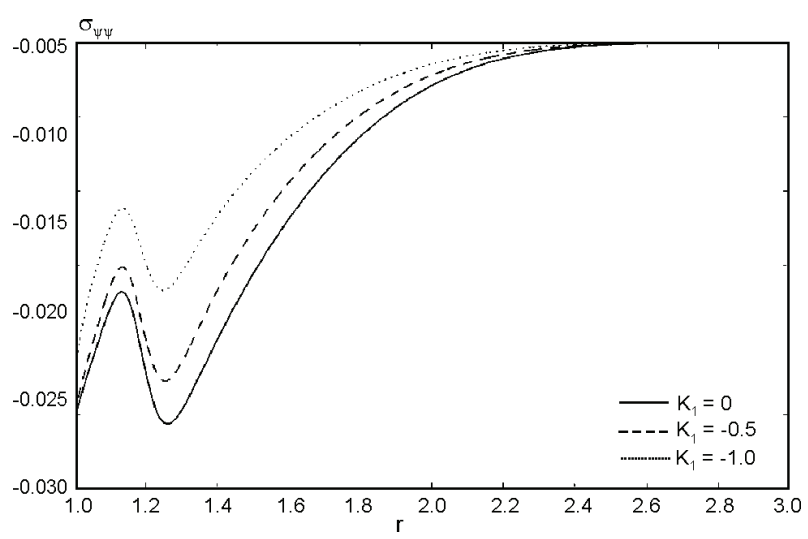

Fig. 4. The stress $\sigma_{\psi \psi}$ distribution with different values of $K_{1}$

progress of all the fields will be more slow and hence the values of all that fields will be decreasing.

\section{CONCLUSION}

Due to this work we can say that, the thermal conductivity plays a very important role in the behavior of the particles of the elastic materials. The consideration of the thermal conductivity to be variable also is very important where all the fields have been affected by this consideration.

\section{References}

[1] M. Biot, Thermoelasticity and irreversible thermo-dynamics, J. Appl. Phys. 127, 240-253 (1956).

[2] H. Lord and Y. Shulman, A generalized dynamical theory of thermoelasticity, Mech. Phys. Solid 15, 299-309 (1967).

[3] A. E. Green and K. A. Lindsay, Thermoelasticity, J. Elast. 2, 1-7 (1972).

[4] S. Erbay and E. Şuhubi, Longitudinal wave propagation in a generalized thermo-elastic cylinder, J. Thermal. Stresses, 9, 279-295 (1986). 
[5] J. Ignaczak, A strong discontinuity wave in thermoelastic with relaxation times, J. Thermal Stresses 8, 25-40 (1985).

[6] J. Ignaczak, Decomposition theorem for thermoelasticity with finite wave speeds, J. Thermal Stresses 1, 41-52 (1978).

[7] M. Ezzat, Fundamental solution in thermoelasticity with two relaxation times for cylindrical regions, Int. J. Eng. Sci. 33, 2011-2020 (1995).

[8] N. M. El-Maghraby and H. M. Youssef, State space approach to generalized thermoelastic problem with thermomechanical shock, J. Applied Mathematics and Computation, 156(2), 577-586 (2004).

[9] N. M El-Maghraby and H. M. Youssef, A two-dimensional thermoelasticity problem for thermo. mechanical shock with two relaxation times, J. Applied Mathematics and Computation (AMC) 120, 172-184 (2005).

[10] H. M. Youssef, N. M. El-Maghraby and A. A. El-Bary, State space approach to thermoelastic problem with vibrational stresses, Computational Mathematics and Modeling 17, 243-253 (2006).

[11] H. Youssef, The Dependence of The Modulus of Elasticity And The Thermal Conductivity on The Reference Temperature in Generalized Thermoelasticity For An Infinite Material With
A Spherical Cavity, Journal of Applied Mathematics and Mechanics, ISSN0253-4827, 26(4) (2005).

[12] H. Youssef, Generalized Thermoelasticity of an Infinite Body with a Cylindrical Cavity and Variable Material Properties, J. Thermal Stresses 28(5), 521-532 (2005).

[13] H. Youssef, Problem of Generalized Thermoelastic Infinite Medium with Cylindrical Cavity Subjected to a Ramp-Type Heating and Loading, J. Archive of App. Mech. 75, 553-565 (2006).

[14] N. Noda, Thermal Stresses in Materials with TemperatureDependent Properties, Thermal Stresses I, Richard B. Hetnarski (Editor), North-Holland, Amsterdam, 1986.

[15] D. P. H. Hasselman and R. A. Heller, Thermal Stresses in Sever Environments, Plenum Press, New York, 1980.

[16] R. B. Hetnaraski, Thermal Stresses, North-Holland, Amsterdam, 1, 391-396 (1986).

[17] O. C. Zienkiewicz and R. L. Taylor, The finite element method. Fluid dynamics, 5th ed. Butterworth-Heinemann, 2000.

[18] J. N. Reddy, An Introduction to the finite element method, second ed., McGraw-Hill, New York, 1993.

[19] R. D. Cook, D. S. Malkus and M. E. Plesha, Concepts and applications of finite element analysis, third ed., John Wiley, New York, 1989.

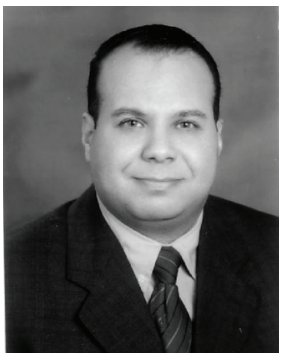

Prof. Hamdy M. Youssef was born on April 22, 1971. In 2003, he received $\mathrm{PhD}$ in Mathematics at Alexandria University, Egypt. His nationality is Egyptian. He is a member of the Egyptian Mathematical Society and IBC Research Council. At present, his affiliation is the Umm Al-Qura University, Saudi Arabia. $\mathrm{He}$ works in the field of theory of thermoelasticity. He has published 25 papers in international journals and 5 conference papers. His most important papers are: Theory of Two-Temperature Generalized Thermoelasticity, IMA Journal of Applied Mathematics, Vol.71(3), pp. 383-390, 2006 and Theory of generalized porothermoelasticity, International Journal of Rock Mechanics and Mining Sciences, Vol. 44, pp. 222-227, 2007. His more detailed CV can be found in "Who's Who in Science and Engineering", 2006-2007 and in "2000 Outstanding Intellectuals of the 21th Century", Cambridge University, 2006.

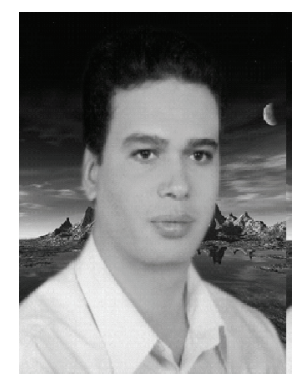

Prof. Ibrahim A. AbBas was born on November 20, 1971. In 2004, he received $\mathrm{PhD}$ in Mathematics at South valley University, Egypt. His nationality is Egyptian. He is a member of the Egyptian Mathematical Society. At present, his affiliation is the Sohag University, Egypt. He works in the field of theory of thermoelasticity and fluid mechanics by finite element method. He has published 15 papers in international journals and 4 conference papers. His most important papers are: "Natural frequencies of a poroelastic hollow cylinder" Acta Mechanica, Vol. 186 1-4, pp. 229-237, 2006, "Finite Element Analysis of Transient Free Convection Flow over Vertical Plate", Forschung im Ingenieurwesen Vol. 70(4), pp. 263-268, 2006 and "Effects of thermal relaxations on thermoelastic interactions in an infinite orthotropic elastic medium with a cylindrical cavity" Archive of Applied Mechanics 2008. His more detailed CV can be found in "Who's Who in Science and Engineering", 2007. 